\title{
Computing large direct products of free groups in integral group rings
}

\author{
Ángel del Río and Manuel Ruiz *
}

\begin{abstract}
We construct explicitly a subgroup of finite minimal index and minimal rank in $\mathbb{Z} G$ which is a direct products of free groups for each finite group $G$ for which this is possible.
\end{abstract}

\section{Introduction and preliminaries}

Let $G$ be a finite group. If $G$ is abelian then the structure of the group of units $\mathbb{Z} G$ of the integral group ring $\mathbb{Z} G$ is well known by the work of Higman, Bass and Milnor. If $G$ is non abelian then generators of subgroups of finite index of $\mathbb{Z} G$ have been found for a large class of groups, (see e.g. [12] and [4]). However the structure of $\mathbb{Z} G$ (or of a subgroup of finite index) is not known except for some few groups. Some of the known cases appeared in [1], [2], [3], [7], [8] and [10].

In a series of three papers ([5], [9] and [6]) Leal, Jespers and the first author characterized the groups $G$ such that $\mathbb{Z} G$ contains a subgroup of finite index which is a direct product of free groups. In those papers the existence of such a large subgroup of $\mathbb{Z} G$ is proved theoretically. Let $G$ be a group satisfying the mentioned property. The aim of these paper is to construct explicitly a subgroup of $\mathbb{Z} G$ which is a direct product of free groups and of minimal index in $\mathbb{Z} G$.

We start with some notation:

The cyclic, dihedral and quaternion groups of order $m$ are denoted by $C_{m}, D_{m}$ and $Q_{m}$ respectively.

Let $G$ be a group and $R$ a ring. Then $R$ denotes the group of units of $R$ and $R G$ the group ring of $G$ with coefficients in $R$. We refer to [12] for

\footnotetext{
${ }^{*}$ The first author has been partially supported by the D.G.I. of Spain and Fundación Séneca of Murcia
} 
notational matters concerning group rings. For any finite subset $H$ of $G$ let $\widehat{H}=\frac{1}{|H|} \sum_{h \in H} h \in \mathbb{Q} G$. More generally, if $\psi: H \rightarrow \mathbb{Z}$ is a map, then we set $\widehat{H_{\psi}}=\frac{1}{|H|} \sum_{h \in H} \psi(h) h$. If $g \in G$, then set $\widehat{g}=\widehat{\langle g\rangle}$. The augmentation map is denoted by $\omega: \mathbb{Z} G \rightarrow \mathbb{Z}$. If $H$ is a normal subgroup of $G$ then $\triangle(G, H)=$ Ker $\omega_{H}$ is the augmentation ideal modulo $H$. We set $\triangle(G)=\triangle(G, G)$. The rank of $G$, denoted by $r(G)$, is the minimum of the cardinalities of the generating subsets of $G$. Set $G^{*}=\operatorname{Hom}(G, \mathbb{Z})$ and let inv $: G \rightarrow G$ denote the map given by $g \mapsto g^{-1}$.

If $K$ is another group and $\varphi: G \rightarrow \operatorname{Aut}(K)$ is a group homomorphism, then $K \rtimes_{\varphi} G$ denotes the corresponding semidirect product.

The non abelian finite groups $G$ such that $\mathbb{Z} G$ contains a subgroup of finite index which is a direct product of free groups, are of the form $G=$ $H \times Z$ where $Z$ is an elementary abelian 2-group and $H$ is of one of the following types (see $[6]$ ):

(a) $\left\langle x, y \mid x^{4}=y^{4}=\left[x^{2}, y\right]=\left[x, y^{2}\right]=[x,[x, y]]=[y,[x, y]]=1\right\rangle$,

(b) $\left\langle x, y_{1}, \ldots, y_{n}\right| x^{4}=y_{i}^{2}=\left[y_{i}, y_{j}\right]=\left[x^{2}, y_{i}\right]=\left[\left[x, y_{i}\right], y_{j}\right]=\left[\left[x, y_{i}\right], x\right]=$ 1),

(c) $\left\langle x, y_{1}, \ldots, y_{n} \mid x^{4}=y_{i}^{4}=y_{i}^{2}\left[x, y_{i}\right]=\left[y_{i}, y_{j}\right]=\left[x^{2}, y_{i}\right]=\left[y_{i}^{2}, x\right]=1\right\rangle$,

(d) $\left\langle x, y_{1}, \ldots, y_{n} \mid x^{2}=y_{i}^{2}=\left[y_{i}, y_{j}\right]=\left[\left[x, y_{i}\right], y_{j}\right]=\left[x, y_{i}\right]^{2}=1\right\rangle$,

(e) $\left\langle x, y_{1}, \ldots, y_{n} \mid x^{2}=y_{i}^{4}=y_{i}^{2}\left[x, y_{i}\right]=\left[y_{i}, y_{j}\right]=\left[\left[x, y_{i}\right], x\right]=1\right\rangle$,

(f) $\left\langle x, y_{1}, \ldots, y_{n} \mid x^{4}=y_{i}^{4}=x^{2} y_{1}^{2}=y_{i}^{2}\left[x, y_{i}\right]=\left[y_{i}, y_{j}\right]=\left[y_{i}^{2}, x\right]=1\right\rangle$,

(g) $\left\langle x, y_{1}, \ldots, y_{n} \mid x^{4}=x^{2} y_{i}^{4}=y_{i}^{2}\left[x, y_{i}\right]=\left[y_{i}, y_{j}\right]=1\right\rangle$,

(h) $U \rtimes_{\phi}\langle x\rangle$ where $U$ is an elementary abelian 3-group, $x$ has order 2 or 4 and $\phi(x)=$ inv.

(i) $U \rtimes_{\phi} K$ where $U$ is an elementary abelian 3-group, $K=\langle x, y\rangle \cong Q_{8}$ and $\phi(x)=\phi(y)=$ inv.

All throughout this paper $G=H \times Z$ where $Z$ is an elementary abelian 2-group and $H$ is of one of the types (a)-(i). The letter $n$ is reserved to denote either the number of $y$ 's for the groups of types (b)-(g) or the rank of $U$ for the groups of type (h) and (i). The rank of $Z$ is always denoted by $k$. 
Our aim is to find a concrete subgroup $F=F_{0} \times \prod_{i=1}^{n} F_{i}$ of finite index of $\mathbb{Z} G$, such that $F_{0}$ is free abelian and $F_{i}$ is free nonabelian and optimal in the sense that the index of $F$ in $\mathbb{Z} G$ is minimal among all the possible subgroups of $\mathbb{Z} G$ which are a direct product of free groups. There is a natural way to obtain such a group if we do not impose the optimal condition. For every primitive central idempotent $e$ of $\mathbb{Q} G$ one of the following conditions holds:

(A) $\mathbb{Q} G \epsilon$ is isomorphic to $\mathbb{Q}$, an imaginary quadratic extension of $\mathbb{Q}$ or a totally definite quaternion algebra over $\mathbb{Q}$.

(B) $\mathbb{Q} G \epsilon$ is a totally definite quaternion algebra over a real quadratic extension of $\mathbb{Q}$.

(C) $\mathbb{Q} G \epsilon$ is isomorphic to $M_{2}(\mathbb{Q})$.

Let $A, B$ and $C$ be the sets of primitive central idempotents of $\mathbb{Q} G$ of types (A), (B) and (C) respectively and $I=A \cup B \cup C$. For every $e \in I$, let $O_{\epsilon}$ be an order in $\mathbb{Q} G e$. Then $O_{e}$ is finite if $e \in A$, virtually infinite cyclic if $e \in B$ and virtually free nonabelian if $e \in C$. Since $O=\prod_{e \in I} O_{e}$ and $\mathbb{Z} G$ are orders in $\mathbb{Q} G$, then $O$ and $\mathbb{Z} G$ are commensurable, that is $O \cap \mathbb{Z} G$ has finite index in both. Therefore $\mathbb{Z} G \cap(1+\mathbb{Q} G \epsilon)$ contains a subgroup of finite index $F_{e}$ which is trivial if $\epsilon \in A$, infinite cyclic if $\epsilon \in B$ and free nonabelian if $\epsilon \in C$. Then $\prod_{e \in I} F_{e}$ is a subgroup of finite index of $\mathbb{Z} G$ with the desired structure. However this does not give information on how big the constructed group is. Surprisingly what we are going to prove in this paper is that this naive approach provides the optimal subgroup in almost all the cases. Our first theorem is:

Theorem 1.1 Let $G=H \times Z$, where $Z$ is an elementary 2-group and $H$ is one of the types (a)-(i). Let $B$ and $C$ be the sets of primitive central idempotents of types $(B)$ and $(C)$ respectively. Then

1. $F_{0}=\mathbb{Z} G \cap\left(1+\mathbb{Q} G f_{B}\right)$, where $f_{B}=\sum_{f \in B} f$, is free abelian of rank $|B|$

2. If $G \neq D_{6}, D_{8}$, then for all $e \in C$

$$
F_{\epsilon}=\mathbb{Z} G \cap(1+\mathbb{Q} G e)
$$

is free non abelian and all the $F_{e}$ 's have the same rank.

3. $F=F_{0} \times \prod_{e \in C} F_{e}$ has finite index in $\mathbb{Z} G$ 
So the group $F=F_{0} \times \prod_{e \in C} F_{e}$ of Theorem 1.1 has the desired properties. We prove Theorem 1.1 in Section 2. Besides we compute the ranks of $F_{0}$ and all the $F_{e}$ 's for all the groups. Moreover every $F_{e}$ is isomorphic to a subgroup of the modular group $S L_{2}(\mathbb{Z})$ that we compute in Proposition 2.1. Using that it is theoretically easy to compute generators for all the $F_{e}$ 's. Also generators of $F_{0}$ are easy to compute by using Proposition 2.3. So there is a method to obtain generators for $F$.

In Section 3 we prove that the group $F$ computed in Section 2 is the best possible. Explicity we prove the following Theorem:

Theorem 1.2 Let $G=H \times Z$ and $F=F_{0} \times \prod_{\epsilon \in C} F_{e}$ as in Theorem 1.1. If $E=E_{0} \times \prod_{j \in J} E_{j}$ is a subgroup of finite index of $\mathbb{Z} G$, where $E_{0}$ is free abelian and $E_{j}$ is free non abelian for every $j$. Then

1. $r\left(E_{0}\right)=|B|$ and $|C|=|J|$.

Besides, if $G \neq D_{8}, D_{6}, Q_{8}$ and $Q_{16}$ then

2. $[\mathbb{Z} G: F] \leq[\mathbb{Z} G: E]$.

3. $r\left(E_{j}\right) \geq r\left(F_{e}\right)$ for every $e \in C$ and $j \in J$.

Note that the exceptional groups $D_{8}, D_{6}, Q_{12}$ and $Q_{16}$ belong to the list of groups we are considering with the following parameters: $k=0$ and $n=1$ for all of them; then $D_{8}$ is of type (d) or (e), $D_{6}$ and $Q_{12}$ are of type (h) with $x$ of order 2 and 4 respectively and finally $Q_{16}$ is of type (g). These four groups have been studied separately in several papers and satisfactory results can be found in [3] for $D_{8} ;[7]$ for $D_{6} ;[10]$ for $Q_{12}$ and [8] for $Q_{16}$.

\section{Large subgroups: Proof of Theorem 1.1}

The statement (3) of Theorem 1.1 follows by the arguments in Section 1. The subsequent Proposition 2.1 implies (2) and Proposition 2.3 implies (1).

For every $a, b \in \mathbb{Z}$ set

$$
\begin{aligned}
\Gamma(b) & =\mathrm{SL}_{2}(\mathbb{Z}) \cap\left[1+b\left(\begin{array}{cc}
\mathbb{Z} & \mathbb{Z} \\
\mathbb{Z} & \mathbb{Z}
\end{array}\right)\right], \\
\Lambda^{a}(b) & =\mathrm{SL}_{2}(\mathbb{Z}) \cap\left[1+b\left(\begin{array}{cc}
\mathbb{Z} & a \mathbb{Z} \\
\mathbb{Z} & \mathbb{Z}
\end{array}\right)\right] \text { and } \\
\Lambda_{a}(b) & =\mathrm{SL}_{2}(\mathbb{Z}) \cap\left[1+b\left(\begin{array}{cc}
a \mathbb{Z} & \mathbb{Z} \\
a \mathbb{Z} & a \mathbb{Z}
\end{array}\right)\right] .
\end{aligned}
$$


If $\Gamma$ is a subgroup of $\mathrm{SL}_{2}(\mathbb{Z})$, then $\widehat{\Gamma}$ denotes the image of $\Gamma$ in $\mathrm{PSL}_{2}(\mathbb{Z})$.

Proposition 2.1 Lete be a primitive central idempotent of $\mathbb{Q} G$ of type $(C)$.

1. Assume that $H$ is of type $(a)-(g)$. Then $G e \simeq D_{8}$ and there are $a, b \in G e$ such that $\{e, a, b, a b\}$ is an integral basis of $\mathbb{Z} G e$. Furthermore if $\alpha e=\alpha_{0} e+\alpha_{1} a+\alpha_{2} b+\alpha_{3} a b$, with $\alpha_{0}, \alpha_{1}, \alpha_{2}, \alpha_{3} \in \mathbb{Z}$ and $\alpha \in \mathbb{Z} G$, then $\omega(\alpha) \equiv \alpha_{0}+\alpha_{1}+\alpha_{2}+\alpha_{3} \bmod 2$.

(i) If $H$ is of type (a) then $e=\left\langle\widehat{\left.x^{2}, y^{2}\right\rangle_{\chi}}\left(1-\widehat{G^{\prime}}\right) \widehat{Z_{\psi}}\right.$ for some non trivial $\chi \in\left\langle x^{2}, y^{2}\right\rangle^{*}$ and some $\psi \in Z^{*}$. Moreover $F_{e} \simeq \Lambda^{2}\left(2^{k+3}\right)$, so that $F_{e}$ is free of rank $1+2^{3 k+6}$,

(ii) Assume that $H$ is of type (b)-(f). For every $i=1, \ldots, n$ let $t_{i}=\left[x, y_{i}\right]$. Let $\phi: G^{\prime} \rightarrow G$ be given by $\phi\left(t_{1}^{i_{1}} t_{2}^{i_{2}} \cdots t_{n}^{i_{n}}\right)=y_{1}^{i_{1}} y_{2}^{i_{2}} \cdots y_{n}^{i_{n}}$. Then

$$
e=e_{S, \varphi, \psi}=\widehat{x^{2}}\left(\widehat{S}-\widehat{G^{\prime}}\right) \widehat{\phi(S)_{\varphi}} \widehat{Z_{\psi}}
$$

where $S$ is a maximal subgroup of $G^{\prime}, \psi \in Z^{*}$ and $\varphi: \phi(S) \rightarrow \mathbb{Z}$ is a map such that $\varphi \circ \phi \in S^{*}$. Moreover, if $H$ is of type $(f)$, then $t_{1} \in S$.

If $H$ is of type (b) or (c), then $F_{e} \simeq \Lambda^{2}\left(2^{2 n+k}\right)$ which is free of rank $1+2^{6 n+3(k-1)}$.

If $H$ is of type (d)-(f) then $F_{e} \simeq \Lambda^{2}\left(2^{2 n+k-1}\right)$. which is free of rank $1+2^{6 n+3(k-2)}$, unless $H$ is of either type (d) or (e), $n=1$ and $k=0$, or equivalently $G \simeq D_{8}$.

(iii) Assume that $H$ is of type (g). For every $i=1, \ldots, n$ let $t_{i}$ be the image of $\left[x, y_{i}\right]$ in $G /\left\langle x^{2}\right\rangle$. Let $\phi: G /\left\langle x^{2}\right\rangle \rightarrow G$ be given by $\phi\left(t_{1}^{i_{1}} t_{2}^{i_{2}} \cdots t_{n}^{i_{n}}\right)=$ $y_{1}^{i_{1}} y_{2}^{i_{2}} \cdots y_{n}^{i_{n}}$. Then

$$
e=e_{S, \varphi, \psi}=\left(\widehat{S}-\widehat{G^{\prime}}\right) \widehat{\phi \pi(S)} \widehat{Z}_{\psi}
$$

where $\pi: G \rightarrow G /\left\langle x^{2}\right\rangle$ is the projection, $S$ is a maximal subgroup of $G^{\prime}$ containing $\left\langle x^{2}\right\rangle, \psi \in Z^{*}$ and $\varphi: \phi \circ \pi(S) \rightarrow \mathbb{Z}$ is a map such that $\varphi \circ \phi \in\left(S /\left\langle x^{2}\right\rangle\right)^{*}$. Moreover $F_{e} \simeq \Lambda^{2}\left(2^{2 n+k}\right)$, so that $F_{e}$ is free of rank $1+2^{6 n+3(k-1)}$

2. Assume that $H$ is of type (h) or (i). Then $G e \simeq D_{6}$ and there exist $a, b \in G e$ such that

$$
\mathbb{Z} G(1-a) e=\mathbb{Z} e(1-a) \oplus \mathbb{Z} a(1-a) \oplus \mathbb{Z} b(1-a) \oplus \mathbb{Z} a b(1-a) .
$$


(i) If $H$ is of type ( $h$ ) with $x$ of order 2 then $e=e_{S \psi}=(\widehat{S}-\widehat{U}) \widehat{Z_{\psi}}$ where $S$ is a maximal subgroup of $U$ and $\psi \in Z^{*}$. Moreover $F_{e} \simeq \Lambda_{3}\left(2^{k} 3^{n-1}\right)$ which is free unless $k=0$ and $n=1$, that is to say, $G=S_{3}$. So if $G \neq S_{3}$ then the rank of $F_{e}$ is $3^{3 n-4}$ if $k=0$, and $2^{3 k-1} 3^{3 n-4}$ otherwise.

(ii) If $H$ is of type $(h)$ with $x$ of order 4 then $e=\epsilon_{S \psi}=\widehat{x^{2}}(\widehat{S}-\widehat{U}) \widehat{Z_{\psi}}$ where $S$ is a maximal subgroup of $U$ and $\psi \in Z^{*}$. Then $F_{e} \simeq \Lambda_{3}\left(2^{k+1} 3^{n-1}\right)$ which is free of rank $1+2^{3 k+2} 3^{3(n-1)}$.

(iii) If $H$ is of type $(i)$, then $e=e_{S, \psi, \chi}=\left(\widehat{S}-\widehat{G^{\prime}}\right) \widehat{Z_{\psi}} \widehat{\langle x y\rangle_{\chi}}$ where $S$ is a maximal subgroup of $U, \psi \in Z^{*}$ and $\chi \in\langle x y\rangle^{*}$. Then $F_{e} \simeq \Lambda_{3}\left(2^{k+2} 3^{n-1}\right)$, which is free of rank $1+2^{3 k+5} 3^{3(n-1)}$.

Proof. The proof is done separately for each case. The first step in each case is to identify the elements of $C$. We leave this part to the reader. Then for each $\epsilon \in C$ one has to make a good selection of $a$ and $b$. This selection induces a ring isomorphism $\phi: \mathbb{Q} G \epsilon \rightarrow M_{2}(\mathbb{Q})$ and using this isomorphism one identifies $\phi\left(F_{\epsilon}\right)$ which happen to be the free subgroup of the modular group in the proposition. Cases (a)-(g) are very similar. We only do case (c) and let the reader check that similar arguments work for the remaining cases. Cases (h) and (i) are also similar so we only do case (i).

Assume that $H$ is of type (c) and let $e=e_{S, \varphi, \psi} \in C$ as in (1.ii). Then $H_{S}=\left\langle x^{2}, G^{\prime}, \phi(S), Z\right\rangle$ is a normal subgroup of index 4 of $G$ and if $t \in G^{\prime} \backslash S$, then $1, x, y=\phi(t), x y$ is a transversal of $G$ modulo $H_{S}$. Set $a=x \epsilon$ and $b=$ ye. Every element $\alpha \in \mathbb{Z} G$ can be written as

$$
\alpha=\beta_{0}+\beta_{1} x+\beta_{2} y+\beta_{3} x y
$$

where $\beta_{i} \in \mathbb{Z} G$ and $\operatorname{Supp}\left(\beta_{i}\right) \subseteq H_{S}$. Moreover if $h \in H_{S}$, then he $= \pm 1$ and therefore $\alpha e=\alpha_{0} e+\alpha_{1} a+\alpha_{2} b+\alpha_{3} a b$ for some $\alpha_{0}, \alpha_{1}, \alpha_{2}, \alpha_{3} \in \mathbb{Z}$ and $\omega(\alpha)=\sum_{i=0}^{3} \omega\left(\beta_{i}\right) \equiv \alpha_{0}+\alpha_{1}+\alpha_{2}+\alpha_{3} \bmod 2$. It is easy to see that $G e \simeq D_{8}$ and $e, a, b, a b$ is an integral basis of $\mathbb{Z} G \epsilon$. Then there is an isomorphism $\rho: \mathbb{Q} G e \rightarrow M_{2}(\mathbb{Q})$ given by

$$
\rho(\alpha e)=\left(\begin{array}{cc}
\alpha_{0}-\alpha_{1}+\alpha_{2}-\alpha_{3} & 2\left(\alpha_{2}-\alpha_{1}\right) \\
\alpha_{1}+\alpha_{3} & \alpha_{0}+\alpha_{1}-\alpha_{2}+\alpha_{3}
\end{array}\right)
$$

(see [3] or [12]). Since the support of $\epsilon$ is $H_{S}, \alpha_{i} \epsilon \in \mathbb{Z} G$ for every $i$ and considering the coefficient of 1 in this element one deduce that $2^{2 n+k} \mid \alpha_{i}$. Let $\gamma_{i}=\alpha_{i} / 2^{2 n+k}$. Then $\alpha=1+2^{2 n+k}\left(\gamma_{0}+\gamma_{1} x+\gamma_{2} y+\gamma_{3} x y\right) e$, so that

$$
\rho(\alpha)=1+2^{2 n+k}\left(\begin{array}{cc}
\gamma_{0}-\gamma_{1}+\gamma_{2}-\gamma_{3} & 2\left(\gamma_{2}-\gamma_{1}\right) \\
\gamma_{1}+\gamma_{3} & \gamma_{0}+\gamma_{1}-\gamma_{2}+\gamma_{3}
\end{array}\right) .
$$


Therefore $\rho(\alpha)=1+2^{2 n+k}\left(\begin{array}{cc}a & 2 b \\ c & d\end{array}\right)$ with $a \equiv d \bmod 2$. Note that this implies that the determinant of $\rho(\alpha)$ is 1 . Thus $\rho\left(F_{e}\right) \subseteq \Lambda_{2}\left(2^{2 n+k}\right)$ and by solving a system of linear equation one can easily verify that the equality holds. Moreover $\Lambda^{2}\left(2^{2 n+k}\right)$ is a subgroup of index 2 of $\Gamma\left(2^{2 n+k}\right)$ and $\Gamma\left(2^{2 n+k}\right) \simeq \Gamma \widehat{\left(2^{2 n+k}\right)}$ is a subgroup of index $2^{6 n+3 k-4}$ of $\widehat{\Gamma(2)}$ (see [11]). Since the last group is free of rank 2 then $\Lambda^{2}\left(2^{2 n+k}\right)$ is free of rank $1+2^{6 n+3(k-1)}$.

Now assume that $H$ is of type (i). Then $e=e_{S, \psi, x}$ as in (2.iii). Set $a=u e$ and $b=x \epsilon$ where $u \in U \backslash S$. Then $H_{S}=\langle x y, S, Z\rangle$ is a normal subgroup of $G$ and $1, u, u^{2}, x, x u, x u^{2}$ is a right transversal of $G$ modulo $H_{S}$. For the first part we argue as in the previous case by noticing that $h e= \pm e$ for every $h \in H_{S}$ and $\left(1+u+u^{2}\right) e=0$. On the other hand $\mathbb{Z} G \epsilon=\mathbb{Z} G(1-a) e$ so that $(1-a) e, a(1-a) e, b(1-a) e, b a(1-a)$ is an integral basis of $\mathbb{Z} G e$. Moreover, as in [12] or [7], there is an isomorphism $\rho: \mathbb{Q} G e \rightarrow M_{2}(\mathbb{Q})$ that associates $\alpha_{0}(1-a) e+\alpha_{1}(1-a) e+\alpha_{2} b(1-a) e+\alpha_{3} b a(1-a) e$ with

$$
\left(\begin{array}{cc}
3\left(\alpha_{0}-\alpha_{1}-\alpha_{2}\right) & -\alpha_{0}+2 \alpha_{1}+2 \alpha_{2}-\alpha_{3} \\
3\left(\alpha_{0}-2 \alpha_{1}-\alpha_{2}-\alpha_{3}\right) & 3\left(\alpha_{1}+\alpha_{2}\right)
\end{array}\right)
$$

Furthermore, if $\alpha \in \mathbb{Z} G \cap(1+\mathbb{Q} G e)=F_{e}$ then $\alpha-1 \in \Delta(G,\langle u\rangle) e=$ $\mathbb{Z} G(1-a) e$ and

$\alpha=1+(\alpha-1) \epsilon=1+\left(\beta_{0}(1-a)+\beta_{1} a(1-a) e+\beta_{2} b(1-a)+\beta_{3} b a(1-a) e\right.$

with $\beta_{i} \in \mathbb{Z}$. Unfortunately in this case, unlike the previous one, the supports of the base elements intersect. However this difficulty can be overcome as follows. The coefficients of $1, u, x$ and $x u$ in $\alpha-1$ are $\gamma_{0}=\frac{\beta_{0}}{2^{k+23^{n-1}}}$, $\gamma_{1}=\frac{\beta_{1}}{2^{k+2} 3^{n-1}}, \gamma_{2}=\frac{\beta_{2}}{2^{k+2} 3^{n-1}}$ and $\gamma_{3}=\frac{\beta_{3}}{2^{k+2} 3^{n-1}}$, respectively. Thus

$$
\rho(\alpha)=1+2^{k+2} 3^{n-1}\left(\begin{array}{cc}
3\left(\gamma_{0}-\gamma_{1}-\gamma_{2}\right) & -\gamma_{0}+2 \gamma_{1}+2 \gamma_{2}-\gamma_{3} \\
3\left(\gamma_{0}-2 \gamma_{1}-\gamma_{2}-\gamma_{3}\right) & 3\left(\gamma_{1}+\gamma_{2}\right)
\end{array}\right)
$$

so that by similar arguments as in the previous paragraph one shows that $\rho\left(F_{\epsilon}\right)=\Lambda_{3}\left(2^{k+2} 3^{n-1}\right)$. The ranks can be computed as in the previous case.

The classical quaternion algebra over an arbitrary ring $R$ is denoted by $\mathbb{H}(R)$.

In order to prove statement (3) of Theorem 1.1 we need information about the central idempotents of $\mathbb{Q} G$ of type (B). This is the role of next Lemma. The proof is straightforward. 
Lemma 2.2 1. If $G$ is not of type (g) or (i) then $B=\emptyset$.

2. Assume that $G$ is of type $(g)$ and let

$$
K=\left\langle y_{1}^{2} y_{2}^{2}, \ldots, y_{1}^{2} y_{n}^{2}, Z\right\rangle=\left\{y_{1}^{2 i_{1}} y_{2}^{2 i_{2}} \ldots y_{n}^{2 i_{n}}: \sum_{t=1}^{n} i_{t} \equiv 0 \bmod 2\right\} \times Z .
$$

For every $\xi \in K^{*}$ and $\epsilon$ every $2 \leq i \leq n$, let $\xi_{n}=\xi\left(y_{1}^{2} y_{i}^{2}\right)$,

$$
K(\xi)=\left\langle y_{1}^{\xi_{2}} y_{2}, \ldots, y_{1}^{\xi_{n}} y_{n}, Z\right\rangle
$$

and

$$
T_{\xi}=\left\{y_{1}^{\sum_{j=2}^{n} \xi_{j} i_{j}} y_{2}^{i_{2}} \ldots y_{n}^{i_{n}}: 0 \leq i_{j} \leq 1\right\} .
$$

Then $T_{\xi}$ is a transversal of $\left\langle x^{2}, K(\xi)\right\rangle$ modulo $K$. Moreover each element of $B$ is of the form

$$
f=f_{\xi, \chi}=\left(1-\widehat{\left.x^{2}\right)} \widehat{K(\xi)}_{\rho \xi, \chi}\right.
$$

for some $\xi \in K^{*}$ and $\chi \in K(\xi)^{*}$ so that $K \subseteq \operatorname{Ker} \chi$ and $\rho_{\xi, \chi}(k t)=\chi(k) \xi(t)$ for every $k \in K$ and $t \in T_{\xi}$. Furthermore

$$
G f_{\xi, \chi}=\left\langle a=x f, b=y_{1} f \mid a^{2}=b^{4}, b^{8}=1, a b a^{-1}=b^{-1}\right\rangle \simeq Q_{16},
$$

$\mathcal{B}=\left\{f, b, b^{2}, b^{3}, a, a b, a b^{2}, a b^{3}\right\}$ is an integral basis of $\mathbb{Z} G f$ and the map $\phi: \mathbb{Q} G f \rightarrow \mathbb{H}(\mathbb{Q}[\sqrt{2}])$ given by

$$
\begin{aligned}
\phi\left(\sum_{g \in \mathcal{B}} \alpha_{g} g\right)= & \alpha_{f}+\frac{\sqrt{2}}{2}\left(\alpha_{b}-\alpha_{b^{3}}\right)+\left(\alpha_{a}+\frac{\sqrt{2}}{2}\left(\alpha_{a b}-\alpha_{a b^{3}}\right)\right) i+ \\
& \left(\alpha_{b^{2}}+\frac{\sqrt{2}}{2}\left(\alpha_{b}+\alpha_{b^{3}}\right)\right) j+\left(\alpha_{a b^{2}}+\frac{\sqrt{2}}{2}\left(\alpha_{a b}+\alpha_{a b^{3}}\right)\right) k
\end{aligned}
$$

is a ring isomorphism.

3. Assume that $G$ is of type (i). Then every element of $B$ is of the form

$$
f_{S, \psi}=\left(1-\widehat{x^{2}}\right)(\widehat{S}-\widehat{U}) \widehat{Z_{\psi}}
$$

where $S$ is a maximal subgroup of $U$ and $\psi \in Z^{*}$ Moreover $G f_{S, \psi} \simeq Q_{12}$ and if $X=x f, Y=y f$ and $W=w f$, with $w \in U \backslash S$, then $\mathcal{B}=$ $\{f, X, Y, X Y, W, W X, W Y, W X Y\}$ is an integral basis of $\mathbb{Z} G f$ and the map $\phi: \mathbb{Q} G f \rightarrow \mathbb{H}(\mathbb{Q}[\sqrt{3}])$ given by

$$
\begin{aligned}
\phi\left(\sum_{g \in \mathcal{B}} \alpha_{g} g\right)= & \frac{1}{2}\left[2 \alpha_{1}-\alpha_{W}+\sqrt{3} \alpha_{X Y W}+\left(2 \alpha_{X Y}-\alpha_{X Y W}-\sqrt{3} \alpha_{W}\right) i\right. \\
& \left.\left(2 \alpha_{X}-\alpha_{X W}-\sqrt{3} \alpha_{Y W}\right) j+\left(2 \alpha_{Y}-\alpha_{Y W}+\frac{\sqrt{3}}{2} \alpha_{X W}\right) k\right]
\end{aligned}
$$

is a ring isomorphism. 
Next proposition provides a proof of statement (3) of Theorem 1.1 and also computes the rank of $F_{0}$.

Proposition 2.3 The group $F_{0}$ is embedded in the centre of $\mathbb{Q} G$. If $H$ is of type $(g)$ then $F_{0}$ is free abelian of rank $2^{2 n+k-2}$; if $H$ is of type (i) then $F_{0}$ is free abelian of rank $2^{k-1}\left(3^{n}-1\right)$. In the remaining cases $F_{0}=1$.

Proof. By Lemma 2.2, we may assume that $G$ is of type (g) or (i). By the comments prior to Theorem 1.1, it is enough to show that for every $f \in B$, $F_{0} f$ is embedded in the centre of $\mathbb{Q} G f$ and realizing that the rank claimed for $F_{0}$ coincides with the cardinality of $B$ that can be easily computed using Lemma 2.2 .

Assume that $H$ is of type $(\mathrm{g})$. Then $f_{B}=1-\widehat{x^{2}}$. If $\alpha \in F_{0}$ then $\alpha-1 \in \Delta\left(G,\left\langle x^{2}\right\rangle\right)$ and hence $(\alpha-1) f_{B} \equiv 0 \bmod 2$, in $\mathbb{Z} G f_{B}$. Therefore, if $f \in B$, then $(\alpha-1) f \equiv 0 \bmod 2$, in $\mathbb{Z} G f$ and, by Lemma $2.2, \phi(\alpha)$ is a unit of $\mathbb{H}(\mathbb{Z}[\sqrt{2}])$, where $\phi$ is the isomorphism of Lemma 2.2. Using that all the units of $\mathbb{H}(\mathbb{Z}[\sqrt{2}])$ are of the form $u, u i, u j$ or $u k$, where $u \in \mathbb{Z}[\sqrt{2}]$, one easily deduces that $\phi(\alpha) \in \mathbb{Z}[\sqrt{2}]$ and hence $\alpha$ is central.

Assume now that $G$ is of type (i). Then $f_{B}=\left(1-\widehat{x^{2}}\right)(1-\widehat{U})$. Therefore, $F_{0} \subseteq \Delta\left(G,\left\langle X^{2}\right\rangle\right) \cap \Delta(G, U)$ and we argue as in the previous case.

\section{Optimality: Proof of Theorem 1.2}

By Theorem [12, Theorem 30.1] the following is a torsionfree normal complement of the trivial units of $\mathbb{Z} G$ :

$$
V=\mathbb{Z} G \cap\left(1+\Delta(G) \Delta\left(G, G^{\prime}\right)\right)
$$

It is well known that $V$ is free nonabelian if $G=D_{8}$ or $G=D_{6}$. Using this fact and Proposition 2.1, it is easy to prove:

Lemma 3.1 For every $e \in C, V e$ is torsionfree.

Set

$$
\widetilde{F}_{0}=\{u \in \mathbb{Z} G: u e=\epsilon \text { for every } e \in C\}
$$

and for every $e \in C$, let

$$
\widetilde{F}_{\epsilon}=\{u \in \mathbb{Z} G: u f=f \text { for all } f \in C \backslash\{e\}\} .
$$

Plainly $\widetilde{F}_{0}=\widetilde{F}_{e_{1}} \cap \widetilde{F}_{e_{2}}$ for every two different $e_{1}$ and $e_{2}$ in $C$. 
The group of type (h) with $n=1, k=0$ and $x$ of order 4 is denoted by $C_{3} \rtimes C_{4}$.

The crux of our argument relies on the following technical lemma.

Lemma 3.2 If $G \neq C_{3} \rtimes C_{4}$ and $G \neq Q_{16}$, then for every $e \in C, \widetilde{F}_{\epsilon} \cap V \subseteq$ $F_{0} \times F_{e}$ and $\widetilde{F}_{0} \cap V \subseteq F_{0}$.

Proof. Let $e \in C$.

Claim 1. If $f$ is a primitive central idempotent, such that $\mathbb{Q} G f$ is commutative or isomorphic to $\mathbb{H}(\mathbb{Q})$, then $V f=f$.

If $\mathbb{Q} G f$ is commutative, then $f\left(1-\widehat{G^{\prime}}\right)=0$ and the claim follows. If $\mathbb{Q} G f \simeq \mathbb{H}(\mathbb{Q})$ then $G f \simeq Q_{8}=\langle a, b\rangle$ and $T=\{f, a, b, a b\}$ is a rational basis of $\mathbb{Q} G f$. Moreover for every $g \in G^{\prime}, g f= \pm f$. This implies that every element of $\Delta(G) \Delta\left(G, G^{\prime}\right) f$ is of the form $2 \alpha f$ for some $\alpha \in \Delta(G)$. Moreover $\alpha f=\sum_{t \in T} \alpha_{t} t$, where $\alpha_{t} \in \mathbb{Z} W, W$ being the kernel of the canonical map $G \rightarrow G f \rightarrow G f /\left\langle a^{2}\right\rangle$. Then $\alpha_{t} f=\beta_{t} f$ where $\beta_{t} \in \mathbb{Z}$ and $\beta_{t} \equiv \omega\left(\alpha_{t}\right) \bmod 2$. Therefore $\sum_{t \in T} \beta_{t} \equiv \omega(\alpha)=0 \bmod 2$. Thus, if $u \in V$, then $u f=1+2 \sum_{t \in T} \beta_{t} t$, with $\sum_{t} \beta_{t}$ even. Since the unique units of $\mathbb{H}(\mathbb{Z})$ are $\pm 1, \pm i, \pm j$ and $\pm i j$, we conclude that $u=f$. This proves Claim 1 .

Claim 2. If $G \neq Q_{12}$ and $f \in A$, then $\left(\widetilde{F}_{\epsilon} \cap V\right) f=f$.

By Claim 1, we may assume that $\mathbb{Q} G f$ is not commutative and $\mathbb{Q} G f \neq$ $\mathbb{H}(\mathbb{Q})$. This implies that $H$ is of type $(h)$ with $x$ of order 4 and $\mathbb{Q} G f$ is isomorphic to the generalized quaternion algebra $A=\mathbb{Q}\left[i, j: i^{2}=-1, j^{2}=\right.$ $-3, i j=k=-j i$. (see [9]). By Proposition 2.1 and [9]

$$
e=\epsilon_{S, \psi}=\widehat{x^{2}}(\widehat{S}-\widehat{U}) \widehat{Z_{\psi}}
$$

and

$$
f=f_{S_{1}, \psi_{1}}=\left(1-\widehat{x^{2}}\right)\left(\widehat{S_{1}}-\widehat{U}\right) \widehat{Z_{\psi}},
$$

where $S$ and $S_{1}$ are two maximal subgroups of $U$ and $\psi, \psi_{1} \in Z^{*}$. Fix $y \in U \backslash S_{1}$. Then $B_{1}=\{f, a=x f, b=y f, a b\}$ is an integral basis of $\mathbb{Z} G f$ and there is an isomorphism $\phi: \mathbb{Q} G f \rightarrow A$ so that $\phi(a)=i$ and $\phi(b)=\frac{1+j}{2}$. Therefore $\phi(\mathbb{Z} G f)$ is the subring of $A$ generated by $i$ and $\frac{1+j}{2}$. Furthermore the only units of this ring are $\pm 1, \pm i, \pm \frac{1-j}{2}, \pm \frac{1+j}{2}, \pm i \frac{1-j}{2}$ and $\pm i \frac{1+j}{2}[10]$.

Let $u \in V \cap \widetilde{F}_{e}$. We have to prove that $u f=f$.

Assume first that $(S, \psi) \neq\left(S_{1}, \psi_{1}\right)$. Let $\epsilon^{\prime}=\epsilon_{S_{1}, \psi_{1}}$. Then $B_{2}=$ $\left\{\epsilon^{\prime}, x \epsilon^{\prime}, y \epsilon^{\prime}, x y \epsilon^{\prime}\right\}$ is an integral basis of $\mathbb{Z} G \epsilon^{\prime}$. Moreover for an $\alpha \in \mathbb{Z} G$ the coefficients of $\alpha f$ and $\alpha \epsilon^{\prime}$ in the basis $B_{1}$ and $B_{2}$ are pairwise congruent modulo 2. Since $(u-1) e^{\prime}=0$, the coefficients of $(u-1) f$ in the basis $B_{1}$ are even. Therefore $u f=1+2\left(\sum_{t \in B_{1}} \alpha_{t} t\right)$ is a unit in $\mathbb{Z} G f$. By the previous 
paragraph $u f= \pm f$. If $u f=-f$, then $(u-1) f=-2 f$. Since $u \in V$, we can write $(u-1) f=\left(\alpha_{0}+\alpha_{1} x\right) \frac{1-x^{2}}{2} \widehat{S_{1}} \widehat{Z_{\psi_{1}}}$ where $\alpha_{0}, \alpha_{1} \in \mathbb{Z} G$ and its support is embedded in a fixed transversal of $G$ modulo $\left\langle x^{2}, S_{1}, Z\right\rangle$ containing 1 . Then the coefficient of 1 in $(u-1) f$ is $\frac{\beta}{2^{k+1} 3^{m-1}}$, where $\beta$ is the coefficient of 1 in $\alpha_{0}$. However the coefficient of $-2 f$ is $-\frac{1}{2^{k} 3^{m}}$, which yields to a contradiction. Thus $u f=f$ as desired.

Now assume that $S=S_{1}$. By Claim 1 and the previous paragraph we have

$$
u-1=(u-1)\left(f_{S, \psi}+\epsilon_{S, \psi}\right)=(u-1)(\widehat{S}-\widehat{U}) \widehat{Z_{\psi}}=\sum_{i=0}^{3} \alpha_{i} x^{i}(\widehat{S}-\widehat{U}) \widehat{Z_{\psi}}
$$

with $\alpha_{i}=\sum_{j=0}^{2} \alpha_{i j} y^{j} \in \Delta\langle y\rangle$. The coefficient of $x^{i} y^{j}$ in $u-1$ is

$$
\frac{3 \alpha_{i j}-\alpha_{i 0}-\alpha_{i 1}-\alpha_{i 2}}{2^{k} 3^{n}}=\frac{\alpha_{i j}}{2^{k} 3^{n-1}} \in \mathbb{Z} .
$$

Therefore $2^{k} 3^{n-1} \mid \alpha_{i j}$ and hence $u f \in f+2^{k} 3^{n-1} \mathbb{Z} G f$ is a unit of $\mathbb{Z} G f$. If $n>1$, then $u f=f$. Assume now that $n=1$. Since we are assuming that $G \neq C_{3} \rtimes C_{4}$, then $k \geq 1$ and we argue as at the end of the previous paragraph to prove that $u f=f$. This finishes the proof of Claim 2 .

Now we prove that $\widetilde{F}_{0} \cap V \subseteq F_{0}$. By Claim 1, the result is obvious unless $H$ is of type (h) and the order of $x$ is 4 . In this case if $G \neq Q_{12}$, then the cardinality of $C$ is greater than 2 and the result is a consequence of Claim 2 .

Now we prove $\widetilde{F}_{\epsilon} \cap V \subseteq F_{0} \times F_{\epsilon}$ for every $\epsilon \in C$. If $H$ is neither of type $(\mathrm{g})$ or (i), then $B=\emptyset$ and hence the Lemma is a direct consequence of Claim 2.

Assume now that $H$ is of type $(\mathrm{g})$ and $G \neq Q_{16}$, so that either $n>1$ or $k>0$. The sum of the elements of $B$ is $f_{B}=1-\widehat{x^{2}}$. Without lost of generality one may assume that

$$
e=\widehat{R}\left(\widehat{S}-\widehat{G^{\prime}}\right) \widehat{Z_{\psi}}
$$

where $R=\left\langle y_{2}, \ldots, y_{n}\right\rangle, S=\left\langle y_{2}^{2}, \ldots, y_{n}^{2}\right\rangle$ and $\psi \in Z^{*}$. The support of $e$ is $L=\left\langle y_{1}^{2}, y_{2}, \ldots, y_{n}\right\rangle \times Z$ and $T_{e}=\left\{1, x, y_{1}, x y_{1}\right\}$ is a transversal of $G$ modulo $L$.

Let $u \in V \cap \widetilde{F}_{e}$ and $\beta=u-1$. If $l \in L$, then $l e= \pm e$. Therefore, $\beta e=\alpha^{\prime} e$ with $\alpha^{\prime} \in \mathbb{Z} G$, Supp $\left(\alpha^{\prime}\right) \subseteq T_{e}$ and $\omega\left(\alpha^{\prime}\right) \equiv \omega(\alpha)=0 \bmod 2$. By Claim 2, $u=1+\alpha^{\prime} e+\beta f_{B}$. It is enough to prove that $\beta_{g}-\beta_{g x^{2}}$ is even for 
every $g \in G$. Indeed, in this case $\beta f_{B} \in \mathbb{Z} G$ and so $u=\left(1+\alpha^{\prime} \epsilon\right)\left(1+\beta f_{B}\right)$, $1+\alpha^{\prime} e \in F_{e}$ and $1+\beta f_{B} \in F_{0}$.

Let $g \in G$ and $t \in T_{e}$ so that $g \equiv t \bmod L$. Then the coefficient of $g$ in $u-1$ is

$$
\pm \frac{\alpha_{t}^{\prime}}{2^{2 n+k}}+\frac{\beta_{g}-\beta_{g x^{2}}}{2}
$$

Therefore $\alpha_{t}^{\prime}=2^{2 n+k-1} \alpha_{t}$ for some $\alpha_{t} \in \mathbb{Z}$ and $\gamma_{g}=\beta_{g}-\beta_{x^{2} g} \equiv \alpha_{t} \bmod 2$. So it is enough to show that $\alpha_{t}$ is even for every $t \in T_{e}$.

To obtain our goal we are going to consider the image of $u f$ under the isomorphism $\phi=\phi_{f}: \mathbb{Q} G f \simeq \mathbb{H}(\mathbb{Q}(\sqrt{2})$, given in Lemma 2.2, for every $f \in$ $B$. Recall that an element of $B$ is of the form $f=f_{\xi, \chi}=\left(1-\widehat{x^{2}}\right) \widehat{K(\xi)_{\rho_{\xi, \chi}}}$ as in Lemma 2.2. We use all the notation of that lemma. Then $\beta f=\sum_{b \in \mathcal{B}} \gamma_{b} b$ where

$$
\gamma_{b}=\sum_{k \in K_{\xi}} \rho_{\xi, \chi}(k)\left(\beta_{b k}-\beta_{b k x^{2}}\right) .
$$

Note that $K(\xi) \cap L$ has index 2 in $K(\xi)$ and hence the cardinality of $K(\xi) \cap L$ is $2^{2(n-1)+k}$. Since either $n>1$ or $k>0$, these cardinality is even. Since $\beta_{g_{1} x^{2}}-\beta_{g_{1} x^{2}} \equiv \beta_{g_{2} x^{2}}-\beta_{g_{2} x^{2}} \bmod 2$ if $g_{1} \equiv g_{2} \bmod L$,

$$
\gamma_{t}=\sum_{k \in K(\xi) \cap L} \rho_{\xi, \chi}(k)\left(\beta_{t h}-\beta_{t h x^{2}}\right)+\sum_{k \in K(\xi) \backslash L} \rho_{\xi, \chi}(k)\left(\beta_{t h}-\beta_{t h x^{2}}\right)
$$

is even. By Lemma $2.2, \phi(\mathbb{Z} G f) \subseteq \mathbb{H}(\mathbb{Z}[\sqrt{2}])$. Since every unit of $\mathbb{H}(\mathbb{Z}[\sqrt{2}])$ is of the form $u, u i, u j$ or $u k$ with $u$ a unit of $\mathbb{Z}[\sqrt{2}]$ then by Lemma 2.2 we deduce that

$$
\gamma_{a}=\gamma_{b^{2}}=\gamma_{a b^{2}}=\gamma_{a b}=\gamma_{a b^{3}}=\gamma_{b}+\gamma_{b^{3}}=0
$$

Writing these equations in terms of the $\beta$ 's we obtain a system of linear equations

$$
\sum_{h \in K(\xi)} \rho_{\xi, \chi}(h)\left(\beta_{t h}-\beta_{t h x^{2}}\right)=0
$$

for every $t=x, y_{1}^{2}, x y_{1}^{2}, x y_{1}, x y_{1}^{3}$. For a fixed $\xi \in K^{*}$ and $k \in K$ the previous system of linear equations becomes

$$
\sum_{t_{1} \in T_{\xi}} \chi\left(t_{1}\right) \sum_{k \in K} \xi(k)\left(\beta_{t t_{1} k}-\beta_{t t_{1} k x^{2}}\right)=0
$$

But the matrix $\left(\chi\left(t_{1}\right)\right)_{t_{1} \in T_{\xi}, \chi \in(K(\xi) / K)^{*}}$ is a Hadamard matrix, that is a matrix of 1 's and -1 's with orthogonal rows. In particular its determinant 
is not zero and hence

$$
\sum_{k \in K} \xi(k)\left(\beta_{t t_{1} k}-\beta_{t t_{1} k x^{2}}\right)=0
$$

for every $\xi \in K^{*}$ and $t_{1} \in K$. Using that the matrix $(\xi(k))_{\xi \in K^{*}, k \in K}$ is a Hadamard matrix too, we deduce that $\beta_{t k}-\beta_{t k x^{2}}=0$ for every $k \in K(\xi)$. Therefore if $g$ is congruent with either $y_{1}^{2}, x, x y_{1}, x y_{1}^{2}$, or $x y_{1}^{3}$ modulo $K(\xi)$ for some $\xi \in K^{*}$ then $\beta_{g}-\beta_{g x^{2}}=0$. In particular $\alpha_{1} \equiv \beta_{y_{1}^{2}}-\beta_{y_{1}^{2} x^{2}}=0 \bmod 2$, $\alpha_{x} \equiv \beta_{x}-\beta_{x^{3}}=0 \bmod 2$ and $\alpha_{x y_{1}} \equiv \beta_{x y_{1}}-\beta_{x^{3} y_{1}}=0 \bmod 2$. Furthermore if $\xi\left(y_{1}^{2} y_{2}^{2}\right)=-1$, then $y_{1}^{-1} y_{2} \in K(\xi)$ and therefore $y_{1} y_{2} \equiv y_{1}^{2} \bmod K(\xi)$. Moreover $y_{1} \equiv y_{1} y_{2} \bmod L$. Thus $\alpha_{y_{1}} \equiv \beta_{y_{1} y_{2}}-\beta_{x^{2} y_{1} y_{2}}=0 \bmod 2$. This finishes the proof for this case.

Assume now that $H$ is of type (i). Then $f_{B}=\left(1-\widehat{x^{2}}\right)(1-\widehat{U})$ and $e=e_{S, \psi}$ as in Proposition 2.1. In order to simplify the arguments we assume that $\psi(z)=1$ for every $z \in Z$. The general case follows by similar arguments. Let $w \in U \backslash S$ and $L=\langle y, S, Z\rangle$. Let $u \in \widetilde{F}_{e} \cap V$. By Claim 2 and Proposition 2.1, $\beta=u-1=\alpha^{\prime} e+\delta f_{B}$ where the support of $\alpha^{\prime}$ is embedded in $\{1, x, w, w x\}$. Then for every $t \in\{1, x\}$ and $l \in L$

$$
\begin{aligned}
& \beta_{t l}=\frac{2 \alpha_{t}^{\prime}-\alpha_{t w}^{\prime}}{2^{k+2} \cdot 3^{n}}+\frac{3^{n} \delta_{t l}-\sum_{v \in U} \delta_{t l v}}{2 \cdot 3^{n}} \\
& \beta_{t w l}=\frac{-\alpha_{t}^{\prime}+2 \alpha_{t w}^{\prime}}{2^{k+2} \cdot 3^{n}}+\frac{3^{n} \delta_{t w l}-\sum_{v \in U} \delta_{t l v}}{2 \cdot 3^{n}} \\
& \beta_{t w^{2} l}=\frac{-\alpha_{t}^{\prime}-\alpha_{t w u}^{\prime}}{2^{k+2} \cdot 3^{n}}+\frac{3^{n} \delta_{t w 2} \sum_{v \in U} \delta_{t l v}}{2 \cdot 3^{n}}
\end{aligned}
$$

where $\delta_{g}=\beta_{g}-\beta_{g x^{2}}$. Thus

$$
\begin{aligned}
2 \alpha_{t}^{\prime}-\alpha_{t w}^{\prime}+2^{k+1}\left(3^{n} \delta_{t l}-\sum_{v \in U} \delta_{t l v}\right) & \equiv \\
-\alpha_{t}^{\prime}+2 \alpha_{t w}^{\prime}+2^{k+1}\left(3^{n} \delta_{t w l}-\sum_{v \in U} \delta_{t l v}\right) & \equiv \\
-\alpha_{t}^{\prime}-\alpha_{t w}^{\prime}+2^{k+1}\left(3^{n} \delta_{t w^{2} l}-\sum_{v \in U} \delta_{t l v}\right) & \equiv 0 \bmod 2^{k+2} 3^{n} .
\end{aligned}
$$

$¿$ From this fact and the equality $\delta_{g}+\delta_{g x^{2}}=0$, for every $g \in G$, one deduces that $2^{k+2} 3^{n}$ divides

$$
2^{k+1}\left(3^{n}\left(\delta_{t l}-\delta_{t x^{2} l}\right)-\sum_{v \in U}\left(\delta_{t l v}-\delta_{t x^{2} l v}\right)\right)=2^{k+2}\left(3^{n} \delta_{t l}-\sum_{v \in U} \delta_{t l v}\right)
$$

for every $t \in\left\{1, x, w, w x, w^{2}, w^{2} x\right\}$ and hence $3^{n}$ divides $\sum_{v \in U} \delta_{g v}$ for every $g \in G$. Then $2^{k+1} 3^{n}$ divides $2 \alpha_{t}^{\prime}-\alpha_{t w}^{\prime}$ and $-\alpha_{t}^{\prime}+2 \alpha_{t w}^{\prime}$ and hence $2^{k+1} 3^{n-1}$ $\alpha_{t}^{\prime}, \alpha_{t w}^{\prime}$. Write $\alpha_{g}^{\prime}=2^{k+1} 3^{n-1} \alpha_{g}$. Then $3 \mid \alpha_{t}+\alpha_{t w}$. Moreover for every $g \in G, \sum_{v \in U} \delta_{g v} \equiv \sum_{w \in\left\langle x^{2}, U\right\rangle} \beta_{g w}=0 \bmod 2$ because $\beta \in \triangle\left(G, G^{\prime}\right)$ and $G^{\prime}=\left\langle x^{2}, U\right\rangle$. Thus

$$
\alpha_{t w} \equiv \delta_{t l} \bmod 2, \alpha_{t} \equiv \delta_{t w l} \bmod 2 \text { and } \alpha_{t}+\alpha_{t w}=\delta_{t w^{2} l} \bmod 2 .
$$


As in the previous case, to finish the proof it is enough to show that $\alpha_{t}$ and $\alpha_{t w}$ are even for every $t=1, x$. Again we obtain our goal by inspection of the isomorphisms $\phi_{f}$ of Lemma 2.2 for $f \in B$. Recall that $\mathcal{B}=\{f, X=$ $x f, Y=y f, X Y, W=w f, X W, Y W, X Y W\}$ is an integral basis of $\mathbb{Z} G f$. Then, by Lemma 2.2 , we have that $\phi(u f)$ is a unit of $\mathbb{Z}\left[\frac{1+\sqrt{3}}{2}, i, j\right]$. The units of this ring are of the form $v$ or $v j$ where $v$ is a unit of $\mathbb{Z}\left[\frac{1+\sqrt{3}}{2}, i\right]$. Moreover $\beta f=\sum_{g \in \mathcal{B}} \gamma_{g} g$, with

$$
\begin{aligned}
& \gamma_{T}=\sum_{z \in Z} \psi(z) \sum_{s \in S}\left(\delta_{t s z}-\delta_{t w^{2} s z}\right) \\
& \gamma_{T W}=\sum_{z \in Z} \psi(z) \sum_{s \in S}\left(\delta_{t w s z}-\delta_{t w^{2} s z}\right)
\end{aligned}
$$

for every $t=f, X, Y, X Y$. Let $t \in\{1, x, y, x y\}$ and $T=t f$. Then

$$
\begin{aligned}
2 \gamma_{T}-\gamma_{T W} & =\sum_{z \in Z} \psi(z) \sum_{s \in S}\left(2 \delta_{t s z}-\delta_{t w s z}-\delta_{t w^{2} s z}\right) \\
& \equiv-\sum_{z \in Z} \psi(z) \sum_{u \in U} \delta_{t z u}=0 \bmod 3 .
\end{aligned}
$$

Thus, by Lemma 2.2, the coefficient of 1 in the expression of $\phi(u f)$ in the basis $\phi(\mathcal{B})$ is not zero and hence $\phi(u f)$ is a unit of $\mathbb{Z}\left[\frac{1+\sqrt{3}}{2}, i\right]$. That is $\gamma_{X}=\gamma_{Y}=\gamma_{X W}=\gamma_{Y W}=0$. In other words

$$
\sum_{z \in Z} \psi(z) \sum_{s \in S}\left(\delta_{t s z}-\delta_{t w^{2} s z}\right)=\sum_{z \in Z} \psi(z) \sum_{s \in S}\left(\delta_{t w s z}-\delta_{t w^{2} s z}\right)=0
$$

for every $t=x, y$. Since the matrix $(\psi(z))_{\psi \in Z^{*}, z \in Z}$ is invertible, then $\sum_{s \in S} \delta_{t s z}-\delta_{t w^{2} s z}=\sum_{s \in S} \delta_{t w s z}-\delta_{t w^{2} s z}=0$ for $t=x, y$ and $z \in Z$. Using these formula and (1) one conclude that

$$
\alpha_{x w} \equiv \alpha_{x} \equiv 3^{n-1} \alpha_{x} \equiv \sum_{s \in S}\left(\delta_{x s z}-\delta_{x w^{2} s z}\right)=0 \bmod 2
$$

and similarly $\alpha_{1} \equiv \alpha_{w} \equiv 0 \bmod 2$, as desired.

We need one more lemma.

Lemma 3.3 If $E$ is a free nonabelian subgroup of $\mathbb{Z} G$ then there is an $e \in C$ such that Ee is nonabelian subgroup. Moreover, for every $\epsilon \in C$ such that Ee is non abelian $\mathrm{Cen}_{\mathbb{Z} G}(E) e \subseteq\{ \pm e\}$.

Proof. If $e \in I \backslash C$ then for every $x, y \in E$, there is $n \geq 1$ so that $\left[(x e)^{n},(y e)^{n}\right]=1$. Therefore there is $e \in C$ so that Ee contains a nonabelian subgroup. Identify $\mathbb{Q} G e$ with $M_{2}(\mathbb{Q})$. Let $a \in \operatorname{Cen}_{\mathbb{Z} G}(E)$. Then $E e$ is a nonabelian subgroup of the centralizer of ae. By considering the canonical 
Jordan form of ae one can prove easily that either ae is central in $\mathbb{Q} G e$ or the centralizer of ae in $M_{2}(\mathbb{Q})$ is abelian. Therefore ae is a unit in the ring of integers of the centre of $M_{2}(\mathbb{Q})$ and hence $a e= \pm e$.

Now we can prove Theorem 1.2. Let $E=E_{0} \times \prod_{j \in J} E_{j}$ be a subgroup of finite index of $\mathbb{Z} G$ so that $E_{0}$ is free abelian and $E_{j}$ is free nonabelian for every $j$. By Lemma 3.3, there is a map $\sigma: J \rightarrow C$ such that for every $j \in J$ there is an $\sigma(j) \in C$ so that $E_{j} \sigma(j)$ is nonabelian and $E_{0} \sigma(j), E_{j_{1}} \sigma(j) \subseteq$ $\{ \pm \sigma(j)\}$, for every $j_{1} \neq j$. This implies that $E_{j} \cap F_{\sigma(j)}$ contains a subgroup of finite index of $F_{\sigma(j)}$. Plainly $\sigma$ is injective. Let now $e \in C$. Then $F_{e} \cap E$ is a subgroup of finite index of $F_{e}$ and therefore $F_{e} \cap E$ is free non abelian. Thus $F_{e} \cap E_{j}$ is non abelian for some $j \in J$ and then $F_{e} \cap E_{j^{\prime}}=1$ for every $j^{\prime} \neq j$. This implies that the cardinality of $C$ and $J$ coincides and $\sigma$ is a bijection. From now on we identify $J$ and $C$ and consider $\sigma$ as an equality, so that for every two different elements $e$ and $f$ of $C, E_{e} e$ is non abelian and $E_{e} f= \pm f$.

Then $E_{0} \cap \widetilde{F}_{0}$ and $F_{0}$ are subgroups of finite index of $\widetilde{F}_{0}$ and therefore they have the same rank. Since the former has also finite index in $E_{0}$, then $r\left(E_{0}\right)=r\left(F_{0}\right)$.

If $a \in E_{e}$, then $a=v g$ for some $v \in V$ and a trivial unit $g \in \pm G$. Then for every $f \in C \backslash\{e\}, \pm f=a f=v f \cdot g f$. Therefore $v f$ is a torsion element of $V f$. By Lemma 3.1, $v f=f$. Combining this with Lemma 3.2 one obtain $E_{e} \subseteq \pm\left(\widetilde{F}_{e} \cap V\right) \rtimes G \subseteq \pm\left(F_{0} \times F_{e}\right) \rtimes G$ and the same argument shows that $E_{0} \subseteq \pm\left(\widetilde{F}_{0} \cap V\right) \rtimes G= \pm F_{0} \rtimes G$. Therefore $E \subseteq F \rtimes( \pm G)$. Since $E$ is torsionfree $[F \rtimes( \pm G): E] \geq 2|G|$ and thus

$$
\begin{aligned}
{[\mathbb{Z} G: E] } & =[\mathbb{Z} G: F \rtimes( \pm G)][F \rtimes( \pm G): E] \\
& \geq[\mathbb{Z} G: F \rtimes( \pm G)] 2|G| \\
& =[\mathbb{Z} G: F \rtimes( \pm G)][F \rtimes( \pm G): F] \\
& =[\mathbb{Z} G: F] .
\end{aligned}
$$

Finally, since $E_{e} \subseteq \pm\left(F_{0} \times F_{e}\right) \rtimes G, E_{\epsilon} \cap F_{0}=1$ and $F_{0}$ is central (Lemma 2.3 , there is an injective homomorphism $f: E_{e} \rightarrow \pm F_{e} \rtimes G$, so that $f\left(E_{e}\right)$ has finite index in $\pm F_{e} \rtimes G$. Then $\left[ \pm F_{e} \rtimes G: F_{e}\right]=2|G| \leq\left[ \pm F_{e} \rtimes G: f\left(E_{e}\right)\right]$ and hence $\left[F_{\epsilon}: F_{e} \cap f\left(E_{e}\right)\right] \geq\left[f\left(E_{e}\right): F_{e} \cap f\left(E_{\epsilon}\right)\right]$. Thus, if $r=r\left(F_{e} \cap f\left(E_{e}\right)\right)$ then

$r\left(F_{e}\right)=1+\frac{r-1}{\left[F_{e}: F_{e} \cap f\left(E_{e}\right)\right]} \leq 1+\frac{r-1}{\left[f\left(E_{e}\right): F_{e} \cap f\left(E_{e}\right)\right]}=r\left(f\left(E_{e}\right)\right)=r\left(E_{e}\right)$. 


\section{References}

[1] P.J. Allen and C. Hobby, A note on the unit group of $\mathbb{Z} S_{3}$, Proc. A.M.S., 99(1) (1987) 9-14.

[2] I. Hugues and K.R. Pearson, The group of units of the integral group ring $\mathbb{Z} S_{3}$, Canadian Math. Bull. 15 (1972) 529-534.

[3] E. Jespers y G. Leal, Describing units in integral group rings of some 2-groups, Comm. Algebra, 19 (1991) 1809-1827.

[4] E. Jespers, Units in integral group rings: a survey. Lect. Notes in Pure and Applied Math. Vol. 198, 141-169, Methods in Ring Theory, Marcel Dekker, New York, 1988.

[5] E. Jespers, G. Leal and . del Río, Products of free groups in the unit group of integral group rings, J. Algebra 180, (1996) 22-40.

[6] E. Jespers and . del Río, A structure theorem for the unit group of the integral group ring of some finite groups, to appear in Journal fr die Reine und Angewandte Mathematik.

[7] E. Jespers and M.M. Parmenter, Bicyclic units in $\mathbb{Z} S_{3}$, Bull. Soc. Math. Belg 44 (1992) 141-145.

[8] E. Jespers and M.M. Parmenter, Units of group rings of groups of order 16, Glasgow Math. J. 35 (1993) 367-379.

[9] G. Leal and . del Río, Products of free groups in the unit group of integral group rings II, J. Algebra 191, (1997) 240-251.

[10] M.M. Parmenter, Free torsion-free normal complements in integral group rings, Comm. Algebra 21 (1993) 3611-3617.

[11] M. Newman, Integral matrices, Academic Press, 1972.

[12] S.K. Sehgal, Units of Integral Group Rings, Longman Scientific and Technical Essex, 1993.

Departamento de Matemáticas, Universidad de Murcia, 30100 Murcia, Spain.

Departamento de Ciencias Jurídicas, Métodos Cuantitativos e Informáticos, Universidad Politécnica de Cartagena, 30203 Cartagena, Murcia, Spain. 